\title{
Concentración óptima de sustancias de bajo costo para mejorar el transporte de dos especies de peces ornamentales
}

\author{
Optimal concentration of low cost substances to improve \\ transportation of two ornamental fish species
}

\section{Concentração ideal de substâncias de baixo custo para melhorar a qualidade de transporte de duas espécies de peixes ornamentais}

\author{
Mónica A. Vásquez - Piñeros ${ }^{1}$, David A. Gómez - B', Wilson F. Ramírez - Duarte ${ }^{3}$, \\ Pedro R. Eslava - Mocha ${ }^{4}$
}

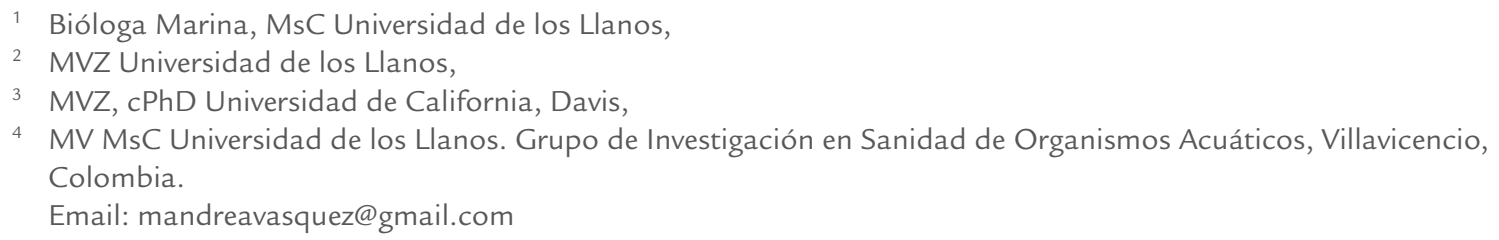

\begin{abstract}
Resumen
Con el propósito de mejorar la calidad del transporte de dos especies de peces ornamentales de interés económico de la región del Orinoco, Paracheirodon axelrodi y Otocinclus sp, se probó el efecto de diferentes concentraciones de sustancias de bajo costo: eugenol $(0.25$ y $0.5 \mathrm{mg} / \mathrm{L})$; cloruro de sodio $(0.5 ; 1.5$ y $2.5 \mathrm{~g} / \mathrm{L})$ y carbonato de calcio $(0,1 ; 0.5$ y $1 \mathrm{~g} / \mathrm{L})$, por dos periodos de transporte (12 y $24 \mathrm{~h}$ ), sobre la calidad de agua, pérdida de iones en el agua, resistencia a eventos de estrés hiperosmótico y sobrevivencia postransporte. La adición de $0.5 \mathrm{mg} / \mathrm{L}$ de eugenol mostró el mejoramiento en la sobrevivencia 7 días posteriores al transporte y una disminución en la pérdida de iones al agua en Otocinclus sp., también mostró una reducción del amonio luego del transporte de 24 h en P.axelrodi. Adicionalmente, la adición de 1.5 g/L de sal resultó efectiva para mejorar la sobrevivencia posterior al desempaque y posterior a los 7 días en ambas especies, y mayor resistencia a la exposición hiperosmótica en $P$. axelrodi. Por último $0.1 \mathrm{~g} / \mathrm{L}$ de carbonato de calcio otorgó una mayor resistencia a eventos de estrés ( $P$. axelrodi) y mejor sobrevivencia de los peces (para ambas especies), a pesar de su poca solubilidad. Se puede concluir que las tres sustancias usadas en concentraciones adecuadas pueden mejorar la calidad del transporte, siendo la sal la sustancia con mayores efectos benéficos sobre la sobrevivencia y la resistencia al estrés, un siguiente paso para la recomendación de estas sustancias, sería evaluar su uso en mezclas para determinar una posible sinergia de estas sustancias.
\end{abstract}

Palabras clave: Otocinclus sp, Paracheroidon axelrodi, eugenol, sal, carbonato de calcio

\begin{abstract}
In order to improve the quality of transport of two ornamental fish species of economic interest in the region of the Orinoco, Paracheirodon axelrodi and Otocinclus sp., the effect of different concentrations of low cost substances: eugenol $(0.25$ and $0.5 \mathrm{mg} / \mathrm{L})$, sodium cloride $(0.5,1.5$ and $2.5 \mathrm{~g} / \mathrm{L})$ and calcium carbonate $(0.1,0.5$ and $1 \mathrm{~g} / \mathrm{L})$ by two periods
\end{abstract}


of transport (12 and $24 \mathrm{~h}$ ), was evaluated on the water quality, ion loss to the water, resistance to hyperosmotic stress and survival after transport and 7 days later. As a result the addition of $0.5 \mathrm{mg} / \mathrm{L}$ of eugenol showed improved survival in 7 days after transport and a decreased ion loss to water in Otocinclus sp. It also reduced ammonium after $24 \mathrm{~h}$ transport in P.axelrodi. Additionally, the addition of $1.5 \mathrm{~g} / \mathrm{L}$ salt was effective to improve survival after unpacking and after 7 days in both species, and greater resistance to hyperosmotic exposure in $P$. axelrodi. Finally, $0.1 \mathrm{~g} / \mathrm{L}$ of calcium carbonate gave a higher resistance to hyperosmotic stress (P. axelrodi) and a better survival for both species, despite its poor solubility. It can be concluded that the three substances used in appropriate concentrations can improve the quality of transport, being the salt the substance with most beneficial effects on survival and stress resistance.

Key words: Otocinclus sp, Paracheroidon alxerodi, eugenol, salt, calcium carbonate

\section{Resumo}

A fim de melhorar a qualidade do transporte de duas espécies de peixes ornamentais de interesse econômico na região do Orinoco, Paracheirodon axelrodi e Otocinclus sp, testou-se o efeito de diferentes concentrações de substâncias de baixo custo: eugenol $(0.25$ e $0.5 \mathrm{mg} / \mathrm{L})$, sal $(0.5,1.5$ e $2.5 \mathrm{~g} / \mathrm{L})$ e carbonato de cálcio $(0.1,0.5$ e 1 g/L) por dois períodos de transporte (12 e 24 h), sobre a qualidade da água, a perda de íons na água, a resistência a estrese hiperósmico e a sobrevivência após o transporte e depois de 7 dias. A adição de $0.5 \mathrm{mg} / \mathrm{L}$ de eugenol melhorou a sobrevivência após 7 dias do transporte e reduziu a perda de íons na água em Otocinclus sp. Também reduziu a amônia após 24 h de transporte em P.axelrodi. Além disso, a adição de $1.5 \mathrm{~g}$ de sal/L foi eficaz para melhorar a sobrevivência ao final do transporte e após 7 dias em ambas espécies, e proporcionou uma maior resistência à exposição hiperósmica em $P$. axelrodi. Finalmente 0.1 $\mathrm{g} / \mathrm{L}$ de carbonato de cálcio deu uma maior resistência ao estresse hiperósmico em $P$. axelrodi e melhor sobrevida para ambas espécies, apesar de sua baixa solubilidade. Pode concluir-se que as três substâncias utilizadas em concentrações adequadas podem melhorar a qualidade do transporte, sendo o sal a substância de com más efeitos benéficos para a sobrevivência e resistência ao estresse.

Palavras chave: Otocinclus sp, Paracheroidon axelrodi, eugenol, sal, carbonato de cálcio

\section{Introducción}

El comercio de peces ornamentales en Colombia ha presentado un crecimiento considerable en las cuatro décadas recientes, soportándose principalmente en la extracción de animales del medio natural. En el año 2009 el 54\% de los peces ornamentales exportados correspondieron a ejemplares de la familia Characidae siendo el cardenal Paracheirodon axelrodi la especie más representativa de esta familia; seguida por la familia Loricariidae con el $20 \%$ de las exportaciones, en la cual el otocinclo Otocinclus sp. fue la especie más exportada (MADR y CCl, 2010). A pesar de su creciente importancia, las actividades de pesca, acopio y comercialización no han desarrollado las bases tecnológicas mínimas para hacerlas más competitivas y sostenibles, lo que se ha manifestado en altas tasas de morbilidad y mortalidad, alteraciones a los ecosistemas y pérdidas económicas importantes. Para los actores de la cadena de peces ornamentales (pescadores, acopiadores, exportadores, productores), el principal problema es la morbilidad y mortalidad que afecta sus costos y la calidad del producto, y a su vez incrementa las tasas de extracción del medio natural. Blanco (1992) reportó que solo se exporta el $42 \%$ de los ejemplares capturados, infiriéndose la existencia de altas tasas de mortalidad y baja calidad de los peces comercializados. En estudios anteriores, se han identificado a los procedimientos de pesca y las primeras fases de transporte como las fases en que se presenta mayor mortalidad y mayor deterioro de los animales (Ramírez-Duarte et al., 2009). De acuerdo con Ramírez y Ajiaco (2001) y Pineda (1996), la tasa de mortalidad varía entre $0 \%$ y $30 \%$, lo que incrementa la extracción de peces de su medio natural hasta en 5.4 millones de ejemplares anuales y a su vez pone en riesgo la sostenibilidad de la actividad.

Con el fin de incrementar la supervivencia, reducir el estrés de los animales, y mejorar la calidad del agua, diversas estrategias han sido evaluadas. Efectos benéficos de la inclusión del cloruro de sodio $(\mathrm{NaCl})$ han sido reportados en varias especies de peces, presentándose variaciones en las concentraciones efectivas de acuerdo con la especie y el tiempo de transporte. Johnson (1979) recomendó una concentración de 2 g/L como guía para el transporte de peces vivos, mientras que Carneiro y Urbinati (2001) demostraron que 6 g/L minimiza el estrés del matrinxã, Brycon amazonicus, durante del transporte. Weirich y Tomasso (1991) recomendaron $11 \mathrm{~g} / \mathrm{L}$ en juveniles de "red drum". De modo semejante, una concentración de sal de $10 \mathrm{~g} / \mathrm{L}$ fue efectiva en el transporte del "striped bass", Morone saxatilis (Walbaum) (Mazik et al., 1991) y de $8 \mathrm{~g} / \mathrm{L}$ en el tambaquí (Colossoma macropomum) durante transportes de hasta $3 \mathrm{~h}$ (Gomes et al., 2003). Sin embargo, en otras especies como Ancistrus triradiatus se han evidenciado efectos benéficos con $2 \mathrm{~g} / \mathrm{l}$ de $\mathrm{NaCl}$ durante el transporte por 12 y $24 \mathrm{~h}$ y $1 \mathrm{~g} / \mathrm{L}$ para transporte de 48 h con (Ramírez-Duarte et al., 2010a, 2010b). Adi- 
cionalmente, la adición de $\mathrm{NaCl}$ incrementa la supervivencia de $A$. triradiatus durante el transporte cuando son sometidos a temperaturas superiores a su rango de confort (Ramírez-Duarte et al ., 2010b).

El uso del eugenol o aceite de clavo, ha sido reportado por Cooke et al., (2004), quienes determinaron que concentraciones entre 5 y $8.5 \mathrm{mg} / \mathrm{L}$ son efectivas para inducir sedación profunda en largemouth bass (Micropterus salmoides) durante 1 h. En matrinxã, Brycon cephalus, una concentración de $5 \mathrm{mg} / \mathrm{L}$ se redujo el estrés durante 4 h y mejoró la calidad del agua (Kioshi et al., 2005). Del mismo modo, las sales de calcio han demostrado un efecto benéfico, aunque moderado, en la reducción del estrés del striped bass (Weirich y Tomaso, 1992) y Brycon amazonicus (Bendhack and Urbinati, 2009) durante el transporte.

Debido a la importancia del cardenal Paracheirodon axelrodi y el otocinclo Otocinclus sp. en el comercio de peces ornamentales en Colombia, este trabajo tiene como objetivo evaluar estrategias de bajo costo que mejoren la calidad del agua y de los animales en el transporte (durante 12 y $24 \mathrm{~h}$ ) mediante el uso de sal común, carbonato de calcio y del anestésico eugenol.

\section{Materiales y métodos}

\section{Animales experimentales}

Se utilizaron lotes de animales silvestres de cardenal (Paracheirodon axelrodi) y otocinclo (Otocinclus sp.), adquiridos de acopiadores locales, se manejaron tallas comerciales de $0.34 \pm 0.44$ y $0.19 \pm 0.08$ g. para Otocinclus sp., y $P$. axelrodi respectivamente. Los animales se aclimataron en acuarios de $40 \mathrm{~L}$, por un periodo no menor a ocho días, teniendo una densidad menor a 1g/L. Los parámetros de calidad de agua se mantuvieron dentro de los rangos óptimos para las especies: temperatura $24{ }^{\circ} \mathrm{C} \pm 1.2, \mathrm{pH} 6.5 \pm 1.1$, sobresaturación de oxígeno, dureza total: $9.13 \pm 2.1 \mathrm{mg} / \mathrm{L}$; alcalinidad total: $4.7 \pm 0.5 \mathrm{mg} / \mathrm{L}$, amoniaco total $0.08 \pm 0.02$ $\mathrm{mg} / \mathrm{L}$. Los animales fueron alimentados dos veces al día con concentrado comercial granulado, con nivel de proteína del $47 \%$, al $1 \%$ de la biomasa total diaria. Siete días después de la recepción de los animales se verificó el estado sanitario del lote, sacrificando y tomando muestras para análisis histopatológico a diez peces de cada lote experimental.

\section{Sustancias experimentales}

Se evaluó la inclusión de tres sustancias de bajo costo, aceite de clavo (eugenol), $\mathrm{NaCl}$ industrial (sin flúor y sin yodo) y carbonato de calcio industrial; durante dos tiempos de transporte de 12 y $24 \mathrm{~h}$. Para cada caso se evaluaron diferentes concentraciones, de la siguiente manera: aceite de clavo: 0.25 y $0.5 \mathrm{mg} / \mathrm{L}$; Sal: $0.5 ; 1.5$ y $2.5 \mathrm{~g} / \mathrm{L}$ y carbonato de calcio con $0.1 ; 0.5$ y $1 \mathrm{~g} / \mathrm{L}$. establecidos de acuerdo a ensayos preliminares.

\section{Condiciones de empaque y transporte}

La alimentación fue suspendida un día antes de iniciar los ensayos. Los animales fueron pesados y posteriormente empacados, con las densidades reportadas por pescadores, acopiadores y exportadores de peces ornamentales locales. La densidad para $P$. axelrodi fue de $18.5 \pm 1.2$ y $11.3 \pm 0.2 \mathrm{~g} / \mathrm{L}$., para transportes de 12 y $24 \mathrm{~h}$ respectivamente y para Otocinclus sp., fue de $27.6 \pm 2.2$ y $19.5 \pm 0.7$ g/L., para los ensayos de 12 y 24 h respectivamente. Se utilizó un volumen de $0.5 \mathrm{~L}$ por bolsa, para disminuir el número de animales experimentales.

El empaque se realizó en doble bolsa plástica con oxígeno en condición de sobresaturación manejando una proporción en volumen agua:oxígeno de 1:3, y cajas de cartón en condiciones de oscuridad. Para registrar la temperatura, se adicionó una bolsa bajo las mismas condiciones de empaque con un termómetro de mínimas y máximas. Las cajas fueron ubicadas en un vehículo, donde se transportaron en 4 y 8 intervalos de 20 minutos aproximadamente, para los ensayos de transporte de 12 y $24 \mathrm{~h}$ respectivamente, permaneciendo en el vehículo durante todo el periodo experimental.

\section{Diseño experimental}

Se llevaron a cabo 6 ensayos para Otocinclus sp., y 6 para $P$. axelrodi en diferentes tiempos. Cada ensayo se llevo a cabo con lotes diferentes de animales y cada uno correspondiente a una sustancia experimental: eugenol (E), sal (S) y carbonato de calcio (CC) y a un periodo de transporte 12 h (12) o 24 h (24). Se realizó tres réplicas por tratamiento. Debido a las diferencias significativas entre los controles de los diferentes ensayos, cada ensayo fue evaluado por separado para determinar las concentraciones óptimas de cada sustancia.

\section{Parámetros de calidad}

Calidad de agua. Una vez cumplidos los periodos de transporte, se abrieron las bolsas, registrando la temperatura y $\mathrm{pH}$ ( $\mathrm{pH}$-meter HI98128, Hanna Instruments); para la determinación de amoniaco total y nitrito las muestras fueron refrigeradas hasta su medición, se de- 
Tabla 1. Diseño experimental de los 6 ensayos de transporte realizados para cada una de las especies Otocinclus sp y P. axelrodi.

\begin{tabular}{|c|c|c|c|c|c|}
\hline \multicolumn{2}{|l|}{ Ensayos } & & \multirow{2}{*}{\multicolumn{3}{|c|}{$\begin{array}{c}\text { Tratamientos } \\
\text { Concentración (Convención) }\end{array}$}} \\
\hline Sustancia & Tiempo (h) & & & & \\
\hline \multirow{2}{*}{ Eugenol (mg/L) } & 12 & control & $0.25(\mathrm{E} 12-0,25)$ & $0.5(\mathrm{E} 12-0.5)$ & - \\
\hline & 24 & control & $0.25(\mathrm{E} 24-0.25)$ & 0.5 (E24-0.5) & - \\
\hline \multirow{2}{*}{ Sal (g/L) } & 12 & control & $0.5(\mathrm{~S} 12-0.5)$ & $1.5(\mathrm{~S} 12-1.5)$ & $2.5(\mathrm{~S} 12-2.5)$ \\
\hline & 24 & control & $0.5(\mathrm{~S} 24-0.5)$ & $1.5(\mathrm{~S} 24-1.5)$ & $2.5(\mathrm{~S} 24-2.5)$ \\
\hline \multirow{2}{*}{ Carbonato de calcio (g/L) } & 12 & control & $0.1(\mathrm{CC} 12-0.1)$ & $0.5(\mathrm{CC} 12-0.5)$ & $1(\mathrm{CC} 12-1)$ \\
\hline & 24 & control & $0.1(\mathrm{CC} 24-0.1)$ & 0.5 (CC24-0.5) & $2(\mathrm{CC} 24-1)$ \\
\hline
\end{tabular}

terminaron mediante el método fotométrico $(\mathrm{HACH}$ DR 2800®), con el Kit TNT plus 831 y el Kit Nitriver$\mathrm{HACH}{ }^{8} 3$, para amoniaco total y nitrito respectivamente. Las concentraciones de amonio se obtuvieron de acuerdo a las fórmulas de Bower y Bidwell (1978) y de Johansson y Wedborg (1980).

lones disueltos en agua. Las concentraciones de sodio y potasio en el agua se determinaron mediante el método de absorción atómica por emisión de llama. La concentración de iones cloruro se determinó mediante el método argenteométrico. Todos los iones fueron reportados como el delta de la concentración en $\mathrm{mg} / \mathrm{L}$, $\Delta=$ concentración final (posterior al desempaque)concentración inicial (anterior al empaque).

Resistencia a concentraciones altas de sal. Se seleccionaron veinte animales al azar de cada tratamiento para la exposición a una solución hiperosmótica (solución de $\mathrm{NaCl}$ de $30 \mathrm{~g} / \mathrm{L}$ ), registrándose el número de animales que conservan el eje de nado cada 5 minutos para cardenal y cada 2 minutos para otocinclo, esto con el fin de determinar la resistencia de los peces después de procedimientos generadores de estrés (Lim et al., 2003). Los animales que perdieron el eje de nado se recuperaron en agua sin sal y oxigenada.

Sobrevivencia. Se registró la sobrevivencia en cada tratamiento inmediatamente finalizado el experimento y a la sobrevivencia acumulada luego de los 7 días posttransporte, teniendo en este caso como animales experimentales aquellos que no fueron utilizados en las pruebas de resistencia a concentraciones altas de sal.

\section{Análisis estadístico}

Las variables de calidad de agua fueron analizadas mediante ANOVA. Posteriormente se verificó la homogeneidad de varianzas mediante la prueba de Levene
$(\mathrm{P}<0,05)$ y la normalidad del error mediante el la prueba Shapiro-Wilk $(\alpha=0.05)$, cuando estos no fueron cumplidos se realizó la prueba no paramétrica de Kruskal-Wallis $(\mathrm{P}<0,05)$. Cuando el de tratamiento fue significativo y los supuestos cumplidos se utilizó la prueba de Tukey $(P<0,05)$ para diferenciar la medias entre las diferentes concentraciones de cada sustancia. Las mortalidades fueron analizadas mediante la prueba Chi cuadrado $(\mathrm{P}<0,05)$. Los resultados de exposición a altas concentraciones de sal fueron evaluados mediante regresión no lineal: En algunos experimentos fue satisfactoria la utilización de modelo exponencial de la forma $Y=a^{*} \exp ^{-b^{*} t}$, donde $Y$ es el número de peces que mantienen el eje de nado en el tiempo $t$, a es el numero de peces inicial y $b$ es la tasa relativa de disminución en el numero de peces que mantiene el eje de nado. En otros experimentos la trayectoria fue sigmoide y se opto por utilizar un modelo logístico de la forma $Y=a$ / $\left(1+\exp ^{-b^{*}\left(c^{*} x\right)}\right)$, donde a es el numero de peces inicial, $b$ la tasa de disminución y c señala el tiempo en que el $50 \%$ de los peces perdió el eje de nado que para este tipo de modelos corresponde al punto de inflexión. La influencia de las concentraciones de cada sustancia sobre los parámetros de estos modelos fue evaluada según la metodología propuesta por Paternoster et al. (1988). Los análisis fueron desarrollados mediante el aplicativo R (R Development Core Team, 2011). Los datos son expresados como media \pm error estándar de la media.

\section{Resultados}

\section{Parámetros de calidad}

Durante el transporte de Otocinclus sp, en los ensayos E12 y E24 no se presentaron diferencias significativas para ninguna de las variables de calidad de agua $(\mathrm{pH}$, amoniaco, amonio y nitritos), tampoco, sin embargo, para iones cloruro y sodio, potasio fueron significativamente menores en los tratamientos E12-0.25; E12- 
0.5; E24-0.25 y E24-0.5 comparados con el control $(\mathrm{P}<0.05)$ (Tabla 2).

Para la misma especie durante los ensayos S12 y S24, no se observaron diferencias significativas en las variables de $\mathrm{pH}$, amoniaco total y amonio. Los niveles de nitrito solo fueron significativamente menores en S241.5 respecto a S24-0.5. Los delta de las concentraciones de sodio del ensayo S12 de todos los tratamientos con sal presentaron valores significativamente menores que el control, al igual que para los ensayos E24 los tratamientos S24-1.5 y S24-2.5. El delta del ion cloruro fue significativamente inferior en los tratamientos S12-0.5 con respecto a S12-2.5. El ion cloruro también disminuyó significativamente en el tratamiento S24-0.5 con respecto a S24-1.5 y a S24-2.5 y el tratamiento S24-1.5 aumentó con respecto al control. El delta de potasio no tuvo diferencias significativas entre los tratamientos de ensayos S12, mientras que en 24 horas los tratamientos S24-0.5; S24-1.5 y S24-2.5 disminuyeron significativamente con respecto al control $(\mathrm{P}<0.05)$ (Tabla 3).

Durante el ensayo CC12 de Otocinclus sp., se observó una mayor concentración de amonio en CC12-0.5 con respecto al control. La pérdida del ion cloruro al agua en CC12-1 fue significativamente menor que el resto de los tratamientos y el control, como también lo fue el tratamiento CC12-0.5 con respecto a CC12-0.1; el valor del delta de sodio en CC12-1 fue significativamente menor que el resto de los tratamientos y el control, mientras que los delta de potasio no tuvieron diferencias significativas en ninguno de los tratamientos. Para el ensayo CC24, la concentración de amoniaco total fue significativamente mayor en el tratamiento CC24-0.5 que en el control, como también fue significativamente mayor el valor de nitrito en CC24-1; el ion cloruro fue significativamente menor en CC24-1 que en el resto de los tratamientos y el control; las concentraciones de sodio fueron significativamente menores en CC24-0.5 y CC24-1. El potasio no presentó diferencias significativas en ninguno de los tratamientos $(\mathrm{P}<0.05)$ (Tabla 4).

Para el caso de $P$. axelrodi, dentro de los ensayos E12, las variables de amoniaco total y iones cloruros no tuvieron diferencias significativas respecto al control, en este ensayo las concentraciones de nitrito en E120.25 y E12-0.5 fueron significativamente menores que el control, los niveles de sodio y potasio tendieron a disminuir con ambas concentraciones, con diferencias significativas en S12-0.25 y S12-0.5 en sodio y S120.25 en potasio, respecto al control. Para los ensayos E24, las concentraciones de amoniaco en E24-0.25 y E24-0.5; y potasio en E24-0.5 fueron significativamente menores en comparación a los controles, para el sodio E24-0.25 fue significativamente menor que el control $(\mathrm{P}<0.05)$ (Tabla 5). El resto de variables no presentaron diferencias significativas.

Para los ensayos de S12 de cardenal se observó una mayor concentración de amonio en S12-2,5 con respecto al control, en el resto de variables de calidad de agua no hubo diferencias significativas; con respecto a los iones, el cloruro presentó una disminución significativa en los tres tratamientos con respecto al control, siendo menor a medida que se aumentó la concentración. Durante los ensayos S24, el pH fue significativamente menor en S24-0.5 y S24-1.5 con respecto al control, el resto de parámetros no tuvo diferencias significativas, mientras que el ion cloruro aumentó significativamente en S24-1.5 y disminuyó significativamente en S24-2.5 con respecto al resto de tratamientos y control, en sodio, solo el tratamiento S24-1.5 fue significa-

Tabla 2. Parámetros de calidad de agua para los ensayos de 12 y $24 \mathrm{~h}$ de eugenol de Otocinclus sp.

\begin{tabular}{|c|c|c|c|c|c|c|c|c|}
\hline Ensayo & $\begin{array}{l}\text { Tto [ ] } \\
\text { (mg/L) }\end{array}$ & $\mathrm{pH}$ & $\begin{array}{c}\text { Amoniaco } \\
\text { total }(\mathrm{mg} / \mathrm{L})\end{array}$ & Amonio $(\mathrm{mg} / \mathrm{L})$ & Nitritos (mg/L) & $\begin{array}{c}\Delta \text { Ion Cloruro } \\
(\mathrm{mg} / \mathrm{L})\end{array}$ & $\Delta$ Sodio $(\mathrm{mg} / \mathrm{L})$ & $\begin{array}{c}\Delta \text { Potasio } \\
(\mathrm{mg} / \mathrm{L})\end{array}$ \\
\hline \multirow{3}{*}{$\mathrm{E} 12^{1}$} & C & $6.61 \pm 0.04$ & $6.73 \pm 0.49$ & $0.0133 \pm 0.0007$ & $0.011 \pm 0.001$ & $18.16 \pm 1.126$ & $5.99 \pm 1.010$ & $1.55 \pm 0.027 \mathrm{a}$ \\
\hline & 0.25 & $6.64 \pm 0.10$ & $6.97 \pm 0.77$ & $0.0096 \pm 0.0024$ & $0.010 \pm 0.003$ & $13.69 \pm 3.852$ & $3.66 \pm 2.882$ & $1.14 \pm 0.198 b$ \\
\hline & 0.5 & $6.59 \pm 0.05$ & $4.90 \pm 0.56$ & $0.0095 \pm 0.0011$ & $0.010 \pm 0.000$ & $7.97 \pm 4.136$ & $4.75 \pm 4.170$ & $1.18 \pm 0.250 b$ \\
\hline \multirow{3}{*}{ E24 } & C & $6.84 \pm 0.07$ & $8.93 \pm 0.87$ & $0.0216 \pm 0.0019$ & $0.009 \pm 0.001$ & $19.46 \pm 3.883$ & $4.56 \pm 1.341$ & $1.63 \pm 0.027 \mathrm{a}$ \\
\hline & 0.25 & $6.78 \pm 0.04$ & $8.33 \pm 0.27$ & $0.0207 \pm 0.0006$ & $0.012 \pm 0.002$ & $11.79 \pm 1.676$ & $2.35 \pm 0.377$ & $0.92 \pm 0.527 b$ \\
\hline & 0.5 & $6.82 \pm 0.08$ & $7.57 \pm 0.43$ & $0.0179 \pm 0.0009$ & $0.010 \pm 0.003$ & $17.85 \pm 0.667$ & $3.57 \pm 0.870$ & $1.52 \pm 0.013 b$ \\
\hline
\end{tabular}

1 La primera letra corresponde al ensayo, de acuerdo a la sustancia experimental, E: eugenol, S: sal y CC: carbonato de calcio, El número corresponde al ensayo de acuerdo al tiempo de transporte $(12 \circ 24 \mathrm{~h})$. Letras diferentes indican diferencias significativas $(\mathrm{P}<0.05)$ entre concentraciones de un mismo ensayo. 
Tabla 3. Parámetros de calidad de agua e iones disueltos en el agua, para los ensayos de 12 y 24 h de sal de Otocinclus sp.

\begin{tabular}{c|c|c|c|c|c|c|c|c}
\hline Ensayo & $\begin{array}{c}\text { Tto [ ] } \\
(\mathbf{g} / \mathbf{L})\end{array}$ & $\mathbf{p H}$ & $\begin{array}{c}\text { Amoniaco } \\
\text { total }(\mathbf{m g} / \mathbf{L})\end{array}$ & Amonio $(\mathbf{m g} / \mathbf{L})$ & Nitritos $(\mathbf{m g} / \mathbf{L})$ & $\begin{array}{c}\Delta \text { lon Cloruro } \\
(\mathbf{m g} / \mathbf{L})\end{array}$ & $\Delta$ Sodio $(\mathbf{m g} / \mathbf{L})$ & $\begin{array}{c}\Delta \mathbf{P o t a s i o} \\
(\mathbf{m g} / \mathbf{L})\end{array}$ \\
\hline & $\mathrm{C}$ & $5.93 \pm 0.09$ & $5.87 \pm 0.81$ & $0.0031 \pm 0.0005$ & $0.0087 \pm 0.0023$ & $14.37 \pm 2.558 \mathrm{ab}$ & $3.19 \pm 0.138 \mathrm{a}$ & $-0.35 \pm 4.447$ \\
& 0.5 & $6.08 \pm 0.06$ & $6.00 \pm 0.95$ & $0.0030 \pm 0.0005$ & $0.0083 \pm 0.0003$ & $0.20 \pm 0.058 \mathrm{~b}$ & $0.11 \pm 0.007 \mathrm{~b}$ & $-5.54 \pm 1.675$ \\
$\mathrm{~S} 12$ & 1.5 & $6.04 \pm 0.03$ & $4.47 \pm 0.32$ & $0.0023 \pm 0.0001$ & $0.0083 \pm 0.0003$ & $26.03 \pm 19.836 \mathrm{ab}$ & $0.07 \pm 0.032 \mathrm{~b}$ & $1.46 \pm 1.722$ \\
& 2.5 & $5.95 \pm 0.05$ & $4.17 \pm 0.23$ & $0.0022 \pm 0.0001$ & $0.0093 \pm 0.0023$ & $32.73 \pm 8.441 \mathrm{a}$ & $0.10 \pm 0.044 \mathrm{~b}$ & $2.82 \pm 2.687$ \\
& $\mathrm{C}$ & $6.34 \pm 0.05$ & $8.07 \pm 1.05$ & $0.0054 \pm 0.0006$ & $0.024 \pm 0.008 \mathrm{ab}$ & $4.83 \pm 10.590 \mathrm{bc}$ & $1.65 \pm 0.240 \mathrm{a}$ & $2.48 \pm 0.084 \mathrm{a}$ \\
& 0.5 & $6.32 \pm 0.11$ & $7.03 \pm 1.15$ & $0.0077 \pm 0.0031$ & $0.032 \pm 0.002 \mathrm{a}$ & $-28.00 \pm 7.095 \mathrm{c}$ & $0.10 \pm 0.035 \mathrm{ab}$ & $-8.23 \pm 0.153 \mathrm{c}$ \\
$\mathrm{S} 24$ & 1.5 & $6.17 \pm 0.08$ & $7.87 \pm 1.31$ & $0.0050 \pm 0.0007$ & $0.010 \pm 0.002 \mathrm{~b}$ & $83.67 \pm 22.244 \mathrm{a}$ & $0.01 \pm 0.049 \mathrm{~b}$ & $-0.43 \pm 0.095 \mathrm{~b}$ \\
& 2.5 & $6.30 \pm 0.05$ & $7.63 \pm 0.42$ & $0.0049 \pm 0.0002$ & $0.0113 \pm 0.0012 \mathrm{ab}$ & $38.67 \pm 5.487 \mathrm{ab}$ & $-0.01 \pm 0.032 \mathrm{~b}$ & $-0.26 \pm 0.328 \mathrm{~b}$ \\
\hline
\end{tabular}

1 La primera letra corresponde al ensayo, de acuerdo a la sustancia experimental, E: eugenol, S: sal y CC: carbonato de calcio, El número corresponde al ensayo de acuerdo al tiempo de transporte $(12 \circ 24 \mathrm{~h})$. Letras diferentes indican diferencias significativas $(P<0.05)$ entre concentraciones de un mismo ensayo.

tivamente mayor que S24-0.5 y para el potasio S24-0.5 fue significativamente mayor comparado con el resto de tratamientos y control $(\mathrm{P}<0.05)$ (Tabla 6).

Durante los ensayos de CC12, no hubo diferencias significativas en relación con las variables de calidad de agua, para los iones cloruro y sodio solo en CC12-1 se observó una disminución significativa de estos iones en el agua, mientras que para potasio se observó un aumento significativo en CC12-0.5 con respecto a los demás grupos. Durante los ensayos CC-24 tampoco se observaron diferencias significativas en la calidad de agua, el ion cloruro y sodio de manera similar disminuyeron en S24-1 con respecto al control (y a los otros tratamientos en el caso del cloruro), en el caso de potasio, este ion solo disminuyó significativamente en CC24-1 con respecto a CC24-0.1 ( $\mathrm{P}<0.05)$ (Tabla 7).

Tabla 4. Parámetros de calidad de agua, iones disueltos en el agua, para los ensayos de 12 y 24 h de carbonato de calcio de Otocinclus sp.

\begin{tabular}{|c|c|c|c|c|c|c|c|c|}
\hline Ensayo & $\begin{array}{c}\text { Tto [ ] } \\
(\mathrm{g} / \mathrm{L})\end{array}$ & $\mathrm{pH}$ & $\begin{array}{c}\text { Amoniaco total } \\
(\mathrm{mg} / \mathrm{L})\end{array}$ & Amonio (mg/L) & Nitritos (mg/L) & $\begin{array}{c}\Delta \text { Ion Cloruro } \\
(\mathrm{mg} / \mathrm{L})\end{array}$ & $\begin{array}{c}\Delta \text { Sodio } \\
(\mathrm{mg} / \mathrm{L})\end{array}$ & $\begin{array}{c}\Delta \text { Potasio } \\
(\mathrm{mg} / \mathrm{L})\end{array}$ \\
\hline \multirow{4}{*}{$\mathrm{CC} 12$} & C & $5.67 \pm 0.07$ & $6.97 \pm 0.19$ & $0.0048 \pm 0.0000 \mathrm{~b}$ & $0.0107 \pm 0.0003$ & $14.02 \pm 1.267 \mathrm{ab}$ & $0.26 \pm 0.183 a$ & $1.63 \pm 0.067$ \\
\hline & 0.1 & $5.99 \pm 0.25$ & $7.34 \pm 0.35$ & $0.0049 \pm 0.0003 \mathrm{ab}$ & $0.0633 \pm 0.0513$ & $15.36 \pm 1.933 a$ & $0.46 \pm 0.136 a$ & $1.71 \pm 0.057$ \\
\hline & 0.5 & $5.79 \pm 0.10$ & $7.91 \pm 0.18$ & $0.0055 \pm 0.0002 \mathrm{a}$ & $0.0403 \pm 0.0278$ & $5.74 \pm 2.953 b$ & $-0.04 \pm 0.090 a$ & $1.77 \pm 0.168$ \\
\hline & 1 & $5.86 \pm 0.17$ & $7.87 \pm 1.15$ & $0.0055 \pm 0.0006 \mathrm{ab}$ & $0.0123 \pm 0.0018$ & $-53.60 \pm 1.097 \mathrm{C}$ & $-2.32 \pm 0.032 b$ & $1.62 \pm 0.065$ \\
\hline \multirow{4}{*}{$\mathrm{CC} 24$} & C & $6.20 \pm 0.13$ & $7.88 \pm 0.71 b$ & $0.0056 \pm 0.0007$ & $0.0090 \pm 0.0006 b$ & $14.89 \pm 1.747 a$ & $0.50 \pm 0.066 b$ & $1.57 \pm 0.081$ \\
\hline & 0.1 & $6.03 \pm 0.08$ & $9.28 \pm 0.93 \mathrm{ab}$ & $0.0067 \pm 0.0007$ & $0.0110 \pm 0.0015 \mathrm{ab}$ & $11.01 \pm 2.495 \mathrm{a}$ & $1.35 \pm 0.058 a$ & $1.67 \pm 0.093$ \\
\hline & 0.5 & $5.85 \pm 0.05$ & $9.67 \pm 0.17 \mathrm{a}$ & $0.0068 \pm 0.0001$ & $0.0123 \pm 0.0018 \mathrm{ab}$ & $5.07 \pm 2.300 \mathrm{a}$ & $-0.06 \pm 0.073 c$ & $1.64 \pm 0.042$ \\
\hline & 1 & $5.90 \pm 0.05$ & $8.75 \pm 0.41 \mathrm{ab}$ & $0.0063 \pm 0.0002$ & $0.0227 \pm 0.0087 a$ & $-54.81 \pm 3.210 b$ & $-2.28 \pm 0.041 d$ & $1.66 \pm 0.035$ \\
\hline
\end{tabular}

1 La primera letra corresponde al ensayo, de acuerdo a la sustancia experimental, E: eugenol, S: sal y CC: carbonato de calcio, El número corresponde al ensayo de acuerdo al tiempo de transporte $(12$ o $24 \mathrm{~h}$ ). Letras diferentes indican diferencias significativas $(P<0.05)$ entre concentraciones de un mismo ensayo. 
Tabla 5. Parámetros de calidad de agua para los ensayos de 12 y $24 \mathrm{~h}$ de eugenol para $P$. axelrodi.

\begin{tabular}{|c|c|c|c|c|c|c|c|c|}
\hline тто & [] $(\mathrm{mg} / \mathrm{L})$ & $\mathrm{pH}$ & $\begin{array}{l}\text { Amoniaco } \\
\text { total }(\mathrm{mg} / \mathrm{L})\end{array}$ & Amonio $(\mathrm{mg} / \mathrm{L})$ & Nitrito $(\mathrm{mg} / \mathrm{L})$ & $\begin{array}{c}\Delta \text { Ion Cloruro } \\
(\mathrm{mg} / \mathrm{L})\end{array}$ & $\triangle$ Sodio $(\mathrm{mg} / \mathrm{L})$ & $\begin{array}{c}\Delta \text { Potasio } \\
(\mathrm{mg} / \mathrm{L})\end{array}$ \\
\hline \multirow{3}{*}{ E12 } & C & $6.17 \pm 0.03$ & $4.67 \pm 0.72$ & $0.0031 \pm 0.0005$ & $0.047 \pm 0.015 a$ & $69.56 \pm 17.360$ & $9.14 \pm 0.457 \mathrm{a}$ & $10.36 \pm 3.203 a$ \\
\hline & 0.25 & $6.19 \pm 0.04$ & $4.97 \pm 0.70$ & $0.0028 \pm 0.0004$ & $0.007 \pm 0.001 b$ & $45.89 \pm 9.035$ & $1.23 \pm 0.623 b$ & $1.43 \pm 0.015 b$ \\
\hline & 0.5 & $6.20 \pm 0.07$ & $3.43 \pm 0.09$ & $0.0021 \pm 0.0001$ & $0.007 \pm 0.003 b$ & $45.25 \pm 11.829$ & $2.19 \pm 2.800 b$ & $3.59 \pm 0.763 \mathrm{ab}$ \\
\hline \multirow{3}{*}{ E24 } & C & $6.23 \pm 0.01$ & $5.10 \pm 0.17 \mathrm{a}$ & $0.0029 \pm 0.0001$ & $0.034 \pm 0.013$ & $28.69 \pm 6.640$ & $6.99 \pm 0.492 a$ & $1.64 \pm 0.166 \mathrm{a}$ \\
\hline & 0.25 & $6.21 \pm 0.10$ & $2.77 \pm 0.52 b$ & $0.0016 \pm 0.0003$ & $0.045 \pm 0.022$ & $17.19 \pm 3.291$ & $-2.47 \pm 0.517 b$ & $1.20 \pm 0.110 \mathrm{a}$ \\
\hline & 0.5 & $6.36 \pm 0.10$ & $2.60 \pm 0.62 b$ & $0.0027 \pm 0.0013$ & $0.050 \pm 0.012$ & $23.59 \pm 3.538$ & $6.75 \pm 0.996 a$ & $-2.66 \pm 0.200 b$ \\
\hline
\end{tabular}

1 La primera letra corresponde al ensayo, de acuerdo a la sustancia experimental, E: eugenol, S: sal y CC: carbonato de calcio, El número corresponde al ensayo de acuerdo al tiempo de transporte $(12 \circ 24 \mathrm{~h})$. Letras diferentes indican diferencias significativas $(\mathrm{P}<0.05)$ entre concentraciones de un mismo ensayo.

Para el caso de Otocinlus sp., en el ensayo E12, los peces de los dos tratamientos presentaron un valor de $b$ (tasa de disminución del número de peces con pérdida del eje de nado) significativamente menor (0.233) que el control $(0.389)(\mathrm{P}<0.05)$. Es decir presentaron una mayor resistencia a la exposición a altas concentraciones de sal. Ambos tratamientos tuvieron la misma trayectoria sin presentar diferencias significativas $(\mathrm{P}<0.05)$. Las relaciones se expresan mediante las siguientes ecuaciones:

$\begin{array}{ll}\text { E12 control: } & Y=20.285^{*} \exp ^{-0.389^{*} \text { tiempo }} R^{2}=0.983 \\ \text { E12-0.25 y E12-0.5: } & Y=20.786^{*} \exp ^{-0.233^{*} \text { tiempo }} R^{2}=0.978\end{array}$

Durante el ensayo E24, de Otocinclus sp., se observó $\boldsymbol{b}$, en E24-0,25 fue la que presentó la tasa más alta de disminución de peces que mantienen el eje de nado (1.6157) que E24-0.5 y que el control (menor resistencia a la exposición a altas concentraciones de sal). Las relaciones se expresan mediante las siguientes ecuaciones:

E24- control: $Y=26.99 /\left(1+\exp ^{-0.7072^{*}(1.479-\text { tiempo })}\right) R^{2}=0.990$ E24-0.5 $\quad \mathrm{Y}=23.94 /\left(1+\exp ^{-0.9778^{*}(1.763-\text { tiempo })}\right) R^{2}=0.985$ E24-0.25: $\quad \mathrm{Y}=22.59 /\left(1+\exp ^{-1.6157^{*}(1.282-\text { tiempo) }}\right) R^{2}=0.998$

En la misma especie para los ensayos $\mathrm{S} 12$, el tratamiento S12-0.5, se caracterizó por presentar la tasa más alta de disminución de peces que mantienen el eje de nado (0.4963), sin embargo esta sólo presento diferencias significativas respecto al tratamiento $\mathrm{S} 12-2.5(\mathrm{P}<0.05)$. Las relaciones expresadas mediante los modelos:

$\begin{array}{ll}\text { S12 control } & Y=20.83^{*} \exp ^{-0.3858^{*} \text { tiempo }} R^{2}=0.970 \\ \text { S12-0.50 } & Y=20.15^{*} \exp ^{-0.4963^{*} \text { tiempo }} R^{2}=0.985 \\ \text { S12-1.5 } & Y=20.51^{*} \exp ^{-0.3788^{*} \text { tiempo }} R^{2}=0.984 \\ \text { S12-2.5: } & Y=21.36^{*} \exp ^{-0.352^{*} \text { tiempo }} R^{2}=0.966\end{array}$

Tabla 6. Parámetros de calidad de agua, iones disueltos en el agua, para los ensayos de 12 y 24 h de sal para $P$. axelrodi.

\begin{tabular}{|c|c|c|c|c|c|c|c|c|}
\hline тाО & $\begin{array}{c}{[\mathrm{l}]} \\
(\mathrm{g} / \mathrm{L})\end{array}$ & pH & $\begin{array}{l}\text { Amoniaco } \\
\text { total }(\mathrm{mg} / \mathrm{L})\end{array}$ & Amonio (mg/L) & Nitrito (mg/L) & $\begin{array}{c}\Delta \text { Ion Cloruro } \\
(\mathrm{mg} / \mathrm{L})\end{array}$ & $\Delta$ Sodio $(\mathrm{mg} / \mathrm{L})$ & $\begin{array}{c}\triangle \text { Potasio } \\
(\mathrm{mg} / \mathrm{L})\end{array}$ \\
\hline \multirow{4}{*}{ S12 } & C & $6.65 \pm 0.29$ & $5.62 \pm 0.07$ & $0.0141 \pm 0.0078 b$ & $0.0110 \pm 0.0006$ & $3.77 \pm 1.884 a$ & $0.31 \pm 0.116 \mathrm{a}$ & $1.58 \pm 0.258 a$ \\
\hline & 0.5 & $6.73 \pm 0.42$ & $5.37 \pm 0.27$ & $0.0130 \pm 0.0070 b$ & $0.0103 \pm 0.0018$ & $-28.00 \pm 0.000 b$ & $-0.48 \pm 0.060 c$ & $1.30 \pm 0.170 \mathrm{a}$ \\
\hline & 1.5 & $6.56 \pm 0.11$ & $5.60 \pm 0.30$ & $0.0072 \pm 0.0021 b$ & $0.0107 \pm 0.0003$ & $-54.00 \pm 11.372 \mathrm{c}$ & $0.03 \pm 0.021 \mathrm{ab}$ & $1.19 \pm 0.082 \mathrm{a}$ \\
\hline & 2.5 & $7.28 \pm 0.20$ & $5.77 \pm 0.09$ & $0.0524 \pm 0.0223 a$ & $0.0107 \pm 0.0019$ & $-421.33 \pm 318.907 c$ & $-0.29 \pm 0.112 b c$ & $0.73 \pm 0.057 b$ \\
\hline \multirow{4}{*}{ S24 } & C & $5.87 \pm 0.06 \mathrm{a}$ & $3.65 \pm 0.16$ & $0.0027 \pm 0.0001$ & $0.0103 \pm 0.0007$ & $-12.73 \pm 4.976 b$ & $0.06 \pm 0.072 a$ & $0.66 \pm 0.076 b$ \\
\hline & 0.5 & $5.50 \pm 0.05 b$ & $3.77 \pm 0.24$ & $0.0027 \pm 0.0001$ & $0.0087 \pm 0.0018$ & $-8.33 \pm 10.667 b$ & $-0.50 \pm 0.035 b$ & $1.02 \pm 0.090 \mathrm{a}$ \\
\hline & 1.5 & $5.51 \pm 0.09 b$ & $3.45 \pm 0.13$ & $0.0024 \pm 0.0001$ & $0.0107 \pm 0.0007$ & $57.67 \pm 24.306 a$ & $0.35 \pm 0.357 a$ & $0.49 \pm 0.012 b$ \\
\hline & 2.5 & $5.69 \pm 0.03 \mathrm{ab}$ & $4.12 \pm 0.17$ & $0.0029 \pm 0.0001$ & $0.0077 \pm 0.0007$ & $-283.67 \pm 209.41 c$ & $-0.06 \pm 0.052 \mathrm{ab}$ & $0.56 \pm 0.032 b$ \\
\hline
\end{tabular}

1 La primera letra corresponde al ensayo, de acuerdo a la sustancia experimental, E: eugenol, S: sal y CC: carbonato de calcio, El número corresponde al ensayo de acuerdo al tiempo de transporte $(12 \circ 24 \mathrm{~h})$. Letras diferentes indican diferencias significativas $(\mathrm{P}<0.05)$ entre concentraciones de un mismo ensayo. 


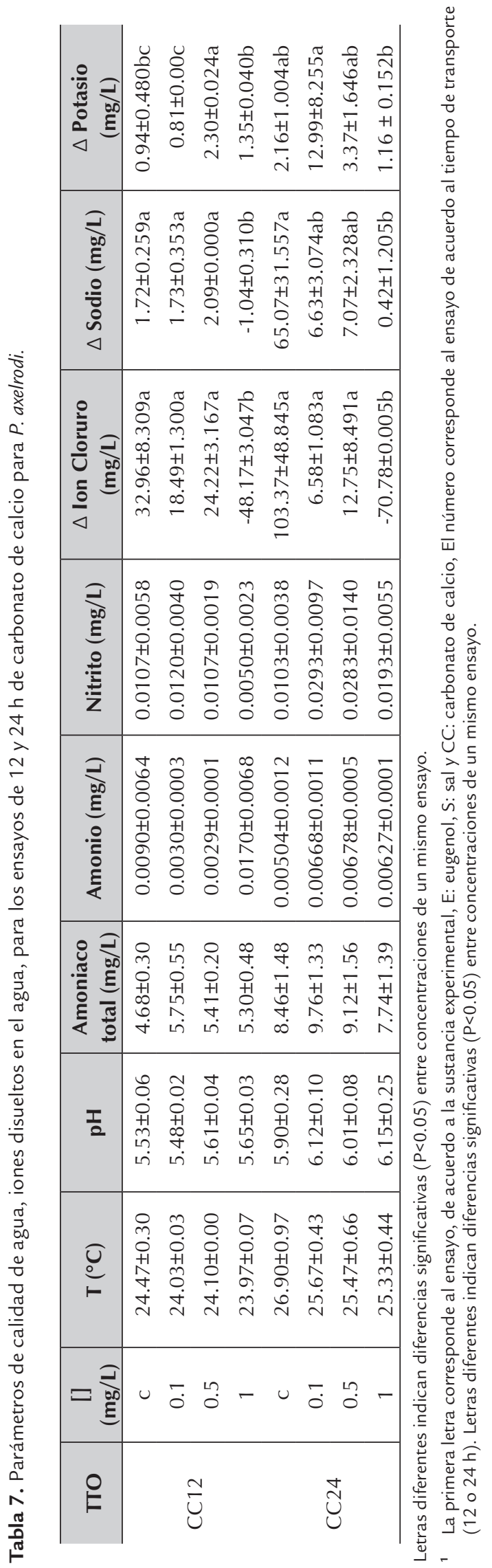


En la evaluación para Otocinclus sp en el ensayo S24, solo el tratamiento S24-0.5 presentó una tasa de disminución significativamente mayor (0.6606) que el control $(0.4476)(\mathrm{P}<0.05)$, el resto de tratamientos no presentaron diferencias significativas con respecto al control y fueron similares entre sí. Los modelos se representaron como:

$\begin{array}{ll}\text { S24 control: } & Y=20.18^{*} \exp ^{-0.4476^{*} \text { tiempo }} R^{2}=0.908 \\ \text { S24- } 0.50: & Y=20.26^{*} \exp ^{-0.6606^{*} \text { tiempo }} R^{2}=0.993 \\ \text { S24-1.5: } & Y=20.15^{*} \exp ^{-0.4728^{*} \text { tiempo }} R^{2}=0.979 \\ \text { S24-2.5: } & Y=19.84^{*} \exp ^{-0.6023^{*} \text { tiempo }} R^{2}=0.985\end{array}$

En los ensayos CC12 con Otocinclus sp., no se presentaron diferencias significativas entre ninguno de los tratamientos, ni tampoco entre los estos y el control $(\mathrm{P}<0.05)$.

CC12 control, CC12-0.1; CC12-0.5 y CC12-1

$\mathrm{Y}=20.84^{\star} \exp ^{-0.5087^{\star} \text { tiempo }} \mathrm{R}^{2}=0.954$

Del mismo modo en CC24 para Otocinclus sp, no se observaron diferencias significativas entre ninguno de los tratamientos y el control $(\mathrm{P}<0.05)$, el modelo que se ajustó a todos los tratamientos fue:

$\mathrm{Y}=20.39^{\star} \exp ^{-0.4897^{*} \text { tiempo }} \mathrm{R}^{2}=0.965$

Para el caso el ensayos de P. axelrodi E12. En este ensayo solo un modelo representó de manera satisfactoria el comportamiento de los tres tratamientos, sin presentar diferencias significativas entre las tasas de disminución de los tratamientos y el grupo control $(\mathrm{P}<0.05)$.

E12-0.25 y E12-0.5 $Y=20.228^{\star} \exp ^{-0.174^{*} \text { tiempo }} R^{2}=0.988$

En esta especie el ensayo E24, el control y el tratamiento E24-0.5 presentaron una disminución ajustada al modelo exponencial y para E-0.25 fue lineal, por lo que no se pueden realizar comparación estadística por ser dos modelos diferentes.
E24 control y E24-0.5 $Y=20.107^{*} \exp ^{-0.249^{*} \text { tiempo }} \quad R^{2}=0.981$ E24-0.25 $Y=20.000-2^{*}$ tiempo $\quad R^{2}=1$

Para el ensayo de cardenal S12, no se observaron diferencias significativas entre el número de peces que perdieron el eje de nado (a) en ninguno de los tratamientos $(\mathrm{P}<0.05)$ (Tabla 8); adicionalmente todos los tratamientos presentaron una tasa de disminución $(\boldsymbol{b})$ superior a la del control (0.1886). Los modelos se presentan como:

$\begin{array}{lll}\text { S12 control } & Y=22.40 /\left(1+\exp ^{-0.1886^{*}(11.09-\text { tiempo })}\right) & R^{2}=0.996 \\ \text { S12-0.5 } & Y=20.88 /\left(1+\exp ^{-0.2979^{*}(11.89-\text { tiempo })}\right) & R^{2}=0.998 \\ \text { S12-1.5 } & Y=20.26 /\left(1+\exp ^{-0.3922^{*}(10.43-\text { tiempo })}\right) & R^{2}=0.999 \\ \text { S12-2.5 } & Y=20.41 /\left(1+\exp ^{-0.4618^{*}(11.79-\text { tiempo })}\right) & R^{2}=0.998\end{array}$

Durante los ensayos S24 de P. axelrodi, no hubo diferencias entre el número de peces que mantuvieron el eje de nado (a) entre los tratamientos; la tasa de disminución $(b)$ fue más significativamente más alta en S24-2.5 en comparación al control y al resto de tratamientos, pero con un tiempo inflexión (c) significativamente menor, es decir menor tiempo para que el $50 \%$ de los peces perdieran el eje de nado (Tabla 9). S24-1.5 se caracterizó por la menor tasa de diminución con diferencias respecto a los demás tratamientos y el control. De manera general las trayectorias del control y de S24-0.5 fueron similares, sin diferencias entre sus parámetros $(\mathrm{P}<0.05)$ (Tabla 9). Las relaciones se presentan como:

$$
\begin{array}{lll}
\text { S24 control } & Y=21.95 /\left(1+\exp ^{-0.2406^{*}(12.94 \text { - tiempo })}\right) & R^{2}=0.976 \\
\text { S24-0.5 } & Y=20.67 /\left(1+\exp ^{-0.2889^{*}(13.11-\text { tiempo })}\right) & R^{2}=0.998 \\
\text { S24-1.5 } & Y=21.99 /\left(1+\exp ^{-0.1875^{*}(15.66 \text { - tiempo })}\right) & R^{2}=0.972 \\
\text { S24-2.5 } & Y=20.53 /\left(1+\exp ^{-0.5542^{*}(8.96-\text { tiempo })}\right) & R^{2}=0.995
\end{array}
$$

Para el ensayo CC12 de cardenal, la tasa de disminución (b) el tratamiento CC12-0.1 se destaco por tener la tasa más alta (0.4223), con diferencias significativas respecto a los demás tratamientos y el control. El parámetro $\boldsymbol{c}$ fue significativamente mayor (es decir posee un punto de inflexión más tardío) en CC12-0.5 com-

Tabla 8. Parámetros del modelo logístico sigmoide $\mathrm{Y}=\mathrm{a} /\left(1+\exp \left(-\mathrm{b}^{*}\left(c^{*} \mathrm{x}\right)\right)\right)$, para $P$. axelrodi, en los ensayos $\mathrm{S} 12$, siendo a, número de peces que mantienen el eje de nado, $\mathbf{b}$ tasa de disminución de número de peces que pierde el eje de nado y $\mathbf{c}$, tiempo en minutos cuando se alcanza el $50 \%$ de $\boldsymbol{a}$.

\begin{tabular}{c|c|c|c|c}
\hline Parámetro & Control & S24-0.5 & S24-1.5 & S24-2.5 \\
\hline $\boldsymbol{A}$ & 22.40 & 20.88 & 20.26 & 20.41 \\
$\boldsymbol{B}$ & $0.1886 \mathrm{c}$ & $0.2979 \mathrm{~b}$ & $0.3922^{\mathrm{a}}$ & $0.4618^{\mathrm{a}}$ \\
$\boldsymbol{C}$ & $11.09 \mathrm{ab}$ & $11.89 \mathrm{a}$ & $10.43^{\mathrm{b}}$ & $11.79^{\mathrm{a}}$ \\
\hline
\end{tabular}


Tabla 9. Parámetros del modelo logístico sigmoide $Y=a /\left(1+\exp \left(-b^{*}\left(c^{*} x\right)\right)\right)$, para $P$. axelrodi, en los ensayos S24, siendo $a$, número de peces que mantienen el eje de nado, $\boldsymbol{b}$ tasa de disminución de número de peces que pierde el eje de nado y $\boldsymbol{c}$, tiempo en minutos cuando se alcanza el $50 \%$ de $\boldsymbol{a}$.

\begin{tabular}{c|r|r|r|r}
\hline Parámetro & \multicolumn{1}{c|}{ Control } & \multicolumn{1}{c|}{ S24-0.5 } & \multicolumn{1}{c|}{ S24-1.5 } & \multicolumn{1}{c}{ S24-2.5 } \\
\hline A & 21.95 & 20.67 & 21.99 & 20.53 \\
B & $0.2406^{\mathrm{bc}}$ & $0.2889^{\mathrm{b}}$ & $0.1875^{\mathrm{c}}$ & $0.5542^{\underline{a}}$ \\
C & $12.94^{\mathrm{a}}$ & $13.11^{\mathrm{a}}$ & $15.66^{\mathrm{a}}$ & $8.96^{\mathrm{b}}$ \\
\hline
\end{tabular}

Tabla 10. Parámetros del modelo logístico sigmoide $Y=a /\left(1+\exp \left(-b^{*}\left(c^{*} x\right)\right)\right)$, para $P$. axelrodi, en los ensayos CC12, siendo a, número de peces que mantienen el eje de nado, $\mathbf{b}$ tasa de disminución de número de peces que pierde el eje de nado y $\mathbf{c}$, tiempo en minutos cuando se alcanza el $50 \%$ de $\boldsymbol{a}$.

\begin{tabular}{c|r|r|r|r}
\hline Parámetro & Control & CC12-0.1 & CC12-0.5 & \multicolumn{1}{c}{ CC12-1 } \\
\hline A & $23.12^{\mathrm{a}}$ & $20.16^{\mathrm{b}}$ & $20.98^{\mathrm{ab}}$ & $30.87^{\mathrm{ab}}$ \\
B & $0.2299^{\mathrm{b}}$ & $0.4223^{\mathrm{a}}$ & $0.2486^{\mathrm{b}}$ & $0.1813^{\mathrm{b}}$ \\
C & $7.90^{\mathrm{c}}$ & $9.33^{\mathrm{ab}}$ & $10.64^{\mathrm{a}}$ & $3.33^{\mathrm{c}}$ \\
\hline
\end{tabular}

parado con el control y con CC12-1. (Tabla 10). Los modelos se describen como:

$\begin{array}{lll}\text { CC12 control } Y=23.12 /\left(1+\exp ^{-0.2299^{*}(7.90-\text { tiempo) })}\right) & R^{2}=0.999 \\ \text { CC12-0.10 } & Y=20.16 /\left(1+\exp ^{-0.4223^{*}(9.33-\text { tiempo) })}\right) & R^{2}=0.997 \\ \text { CC12-0.5 } & Y=20.98 /\left(1+\exp ^{-0.2486^{*}(10.64-\text { tiempo) })}\right) & R^{2}=0.995 \\ \text { CC12-1 } & Y=30.87 /\left(1+\exp ^{-0.1813^{*}(3.33-\text { tiempo) })}\right) & R^{2}=0.997\end{array}$

En el ensayo CC24, con relación a la tasa de disminución $\boldsymbol{b}$, el tratamiento CC24-0.1 no tuvo diferencias significativas con el control, pero si fue significativamente mayor en comparación con CC24-0.5 y CC24-1, por el contrario el tratamiento CC24-1 presentó una tasa menor de disminución comparada con CC24-0.5 y CC24-1. El tiempo de inflexión c no presentó diferencias entre tratamientos $(\mathrm{p}<0.05)$ (Tabla 11).

\section{Sobrevivencia}

Para Otocinclus sp., no se observaron diferencias significativas en sobrevivencia postransporte en los ensayos E12 y E24, pero la sobrevivencia posterior a los 7 días fue significativamente mayor en E12-0.25 y E12-0.5 que el control (Tabla 11). En los ensayos S12 se presentaron diferencias significativas postransporte en S12-1.5 y S12-2.5 respecto al resto de tratamientos, en los ensayos S24 la sobrevivencia da luego de 7 días fue significativamente mayor en S241.5 que el control (Tabla 12). El ensayo de CC12 solo presentó diferencias significativas en la sobrevivencia post-transporte 7 días, para CC12-0.1 con respecto al control (Tabla 13).

Tabla 11. Parámetros del modelo logístico sigmoide $\mathrm{Y}=\mathrm{a} /\left(1+\exp \left(-\mathrm{b}^{*}\left(c^{*} \mathrm{x}\right)\right)\right)$, para $P$. axelrodi, en los ensayos CC24, siendo a, número de peces que mantienen el eje de nado, $\mathbf{b}$ tasa de disminución de número de peces que pierde el eje de nado y $\mathbf{c}$, tiempo en minutos cuando se alcanza el $50 \%$ de $\boldsymbol{a}$.

\begin{tabular}{c|r|r|r|r}
\hline Parámetro & \multicolumn{1}{c|}{ Control } & \multicolumn{1}{c|}{ CC24-0.1 } & \multicolumn{1}{c|}{ CC24-0.5 } & \multicolumn{1}{c}{ CC24-1 } \\
\hline A & $22.71^{\mathrm{b}}$ & $21.17^{\mathrm{b}}$ & $23.12^{\mathrm{b}}$ & $33.22^{\mathrm{a}}$ \\
B & $0.252^{\mathrm{abc}}$ & $0.3551^{\mathrm{a}}$ & $0.2299^{\mathrm{c}}$ & $0.1402^{\mathrm{b}}$ \\
C & 7.48 & 8.11 & 7.9 & 2.89 \\
\hline
\end{tabular}


Tabla 12. Porcentaje de sobrevivencia post-transporte (\%PT) y posterior a los 7 días (\%7D) de Otocinclus sp, en los tratamientos de los ensayos de eugenol durante el transporte de 12 y 24 horas

\begin{tabular}{c|c|c|c}
\hline & [] $\mathbf{~ m g / L}$ & \multicolumn{1}{c}{ \% PT } & \multicolumn{1}{c}{ \% 7D } \\
\hline \multirow{7}{*}{12 horas } & C & $94.29 \pm 2.96$ & $87.22 \pm 3.16 \mathrm{a}$ \\
& 0.25 & $98.29 \pm 2.96$ & $97.85 \pm 3.72 \mathrm{~b}$ \\
& 0.5 & $97.43 \pm 2.56$ & $100 \pm 0.0 \mathrm{~b}$ \\
& $\mathrm{C}$ & $97.78 \pm 1.92$ & $98.48 \pm 2.62$ \\
& 0.25 & $98.89 \pm 1.92$ & $91.66 \pm 2.89$ \\
& 0.5 & $100 \pm 0.0$ & $98.48 \pm 2.62$ \\
\hline
\end{tabular}

Tabla 13. Porcentaje de sobrevivencia post-transporte (\%PT) y posterior a los 7 días (\%7D) de Otocinclus sp, en los tratamientos de los ensayos de sal durante el transporte de 12 y 24 horas

\begin{tabular}{c|c|c|c}
\hline & {[] $\mathbf{g} / \mathbf{L}$} & \% PT & \% 7D \\
\hline \multirow{4}{*}{12} & $\mathrm{C}$ & $83.63 \pm 9.37^{\mathrm{a}}$ & $89.64 \pm 3.37$ \\
horas & 0.5 & $84.59 \pm 7.92^{\mathrm{a}}$ & $87.69 \pm 16.48$ \\
& 1.5 & $93.19 \pm 3.12^{\mathrm{b}}$ & $93.43 \pm 6.42$ \\
& 2.5 & $94.22 \pm 3.88^{\mathrm{b}}$ & $93.28 \pm 2.95$ \\
& $\mathrm{C}$ & $92.86 \pm 7.14$ & $88.32 \pm 2.92 \mathrm{a}$ \\
\multirow{2}{*}{24} & 0.5 & $96.43 \pm 3.57$ & $90.76 \pm 0.25 \mathrm{ab}$ \\
& 1.5 & $97.62 \pm 4.12$ & $98.49 \pm 2.62 \mathrm{~b}$ \\
& 2.5 & $94.10 \pm 2.06$ & $96.39 \pm 3.13 \mathrm{ab}$ \\
\hline
\end{tabular}

Para el caso de cardenal, en los ensayos E12 y E24, ninguno de los tratamientos presentaron diferencias significativas entre sí, ni diferencias con los controles (Tabla 14). En los ensayos S12 los tres tratamientos presentaron sobrevivencias mayores al control. La sobrevivencia postransporte 7 días en este ensayo fue menor en S12-2,5 en que en S12-1.5, el resto de tratamientos para esta variable no tuvo diferencias significativas. Durante el ensayo S24 no hubo diferencias significativas entre los tratamientos y controles (Tabla 15). En el ensayo CC12, no se presentaron diferencias significativas en ninguno de los tratamientos, mientras que para el ensayo CC24, el tratamiento CC24-0.1 presentó una sobrevivencia mayor al control y a CC24-1 $(\mathrm{P}<0.05)$ (Tabla 16).
Tabla 14. Porcentaje de sobrevivencia post-transporte (\%PT) y posterior a los 7 días (\%7D) de Otocinclus sp, en los tratamientos de los ensayos de carbonato de calcio en el transporte de 12 y 24 horas

\begin{tabular}{c|c|c|c}
\hline & {[] $\mathbf{~ g / L}$} & \% PT & \% 7D \\
\hline \multirow{4}{*}{12} & $\mathrm{C}$ & $96.74 \pm 2.27$ & $80 \pm 3.58 \mathrm{a}$ \\
horas & 0.1 & $98.78 \pm 1.06$ & $297.79 \pm 2.22 \mathrm{~b}$ \\
& 0.5 & $97.50 \pm 0.89$ & $93.09 \pm 0.80 \mathrm{a}$ \\
& 1 & $95.92 \pm 3.53$ & $85.47 \pm 4.40 \mathrm{a}$ \\
& $\mathrm{C}$ & $98.37 \pm 2.82$ & $88.20 \pm 10.28$ \\
horas & 0.1 & $95.61 \pm 2.7$ & $91.77 \pm 1.68$ \\
& 0.5 & $94.59 \pm 2.77$ & $83.35 \pm 3.24$ \\
& 1 & $95.28 \pm 2.93$ & $85.73 \pm 6.02$ \\
\hline
\end{tabular}

Tabla 15. Porcentaje de sobrevivencia post-transporte (\%PT) y posterior a los 7 días (\%7D) de P. axelrodi, en los tratamientos de los ensayos de eugenol durante el transporte de 12 y 24 horas

\begin{tabular}{c|c|r|r}
\hline & [] $\mathbf{~ m g / L}$ & \multicolumn{1}{c|}{ \% PT } & \multicolumn{1}{c}{ \% 7D } \\
\hline \multirow{4}{*}{12 horas } & $\mathrm{C}$ & $91.33 \pm 5.77$ & $97.73 \pm 0.0$ \\
& 0.25 & $95 \pm 4.16$ & $96 \pm 0.15$ \\
& 0.5 & $95 \pm 4.16$ & $96 \pm 5.04$ \\
& $\mathrm{C}$ & $95.56 \pm 1.92$ & $92.13 \pm 6.85$ \\
& 0.25 & $97.78 \pm 1.92$ & $96.10 \pm 3.85$ \\
& 0.5 & $95.56 \pm 1.92$ & $94.32 \pm 2.3$ \\
\hline
\end{tabular}

Tabla 16. Porcentaje de sobrevivencia post-transporte (\%PT) y posterior a los 7 días (\%7D) de P.axelrodi, en los tratamientos de los ensayos de sal durante el transporte de 12 y 24 horas

\begin{tabular}{c|c|c|c}
\hline & {[] $\mathbf{~ g / L}$} & \%PT & \% 7D \\
\hline \multirow{4}{*}{12} & $\mathbf{C}$ & $93.75 \pm 10.83 \mathrm{a}$ & $85.86 \pm 17.23 \mathrm{ab}$ \\
horas & 0.5 & $99.32 \pm 1.18 \mathrm{~b}$ & $91.34 \pm 8.35 \mathrm{ab}$ \\
& 1.5 & $100 \pm 0.0 \mathrm{~b}$ & $97.62 \pm 4.120 .0 \mathrm{~b}$ \\
& 2.5 & $100 \pm 0.0 \mathrm{~b}$ & $85.55 \pm 6.0 \mathrm{a}$ \\
& $\mathrm{C}$ & $98.85 \pm 1.99$ & $96.97 \pm 5.25$ \\
24 & 0.5 & $0.0 \pm 0.0 \mathrm{~b}$ & $0.0 \pm 0.0$ \\
horas & 1.5 & $0.0 \pm 0.0 \mathrm{~b}$ & $0.0 \pm 0.0$ \\
& 2.5 & $1.11 \pm 1.92$ & $0.0 \pm 0.0$ \\
\hline
\end{tabular}


Tabla 17. Porcentaje de sobrevivencia post-transporte (\%PT) y posterior a los 7 días (\%7D) de P.axelrodi, en tratamientos de los ensayos de carbonato de calcio en el transporte de 12 y 24 horas

\begin{tabular}{|c|c|r|r|}
\hline \multicolumn{1}{c|}{} & {$[\mathbf{~ g} / \mathbf{L}$} & \multicolumn{1}{c|}{ \%PT } & \multicolumn{1}{c|}{ \% 7D } \\
\hline \multirow{4}{*}{$\begin{array}{c}12 \\
\text { horas }\end{array}$} & $\mathbf{C}$ & $99.29 \pm 1.23$ & $99.21 \pm 1.32$ \\
\cline { 2 - 4 } & 0.1 & $100 \pm 0.0$ & $100 \pm 0.0$ \\
\cline { 2 - 4 } & 0.5 & $98.61 \pm 2.4$ & $96.76 \pm 3.37$ \\
\cline { 2 - 4 } & 1 & $99.32 \pm 1.18$ & $099.21 \pm 1.37$ \\
\hline \multirow{3}{*}{$\begin{array}{c}24 \\
\text { horas }\end{array}$} & $\mathbf{C}$ & $95.55 \pm 5.09 \mathrm{bc}$ & $98.48 \pm 2.62$ \\
\cline { 2 - 4 } & 0.1 & $77.55 \pm 1.53 \mathrm{a}$ & $97.92 \pm 3.61$ \\
\cline { 2 - 4 } & 0.5 & $88.89 \pm 5.09 \mathrm{ab}$ & $98.15 \pm 3.21$ \\
\cline { 2 - 4 } & 1 & $97.9 \pm 1.85 \mathrm{c}$ & $100 \pm 0.0$ \\
\hline
\end{tabular}

\section{Discusión}

\section{Adición de eugenol en el transporte}

Otocinclus sp. La utilización de eugenol en concentraciones de 0.25 y $0.5 \mathrm{mg} / \mathrm{L}$, en los ensayos de transporte de Otocinclus sp., no tuvo efectos significativos sobre la calidad de agua. Debido a que tampoco hubo diferencias significativas en la mortalidad postransporte, se infiere que estas bajas concentraciones de anestésico no lograron reducir la tasa metabólica y por tanto la excreción de residuos nitrogenados (Cooke et al., 2004).

A pesar de no observar diferencias significativas en calidad de agua y la mortalidad postransporte en este ensayo, la incorporación de $0.5 \mathrm{mg} / \mathrm{L}$ de eugenol otorgó una resistencia a altas exposiciones de sal significativamente mayor en los dos periodos de transporte y una mejor sobrevivencia posterior a los 7 días en transporte de $12 \mathrm{~h}$ con 0.25 y $0.5 \mathrm{mg} / \mathrm{L}$. De manera similar, Kioshi et al. (2005) reportó reducción del estrés en Brycon cephalus, durante $4 \mathrm{~h}$ a una concentración de $5 \mathrm{mg} / \mathrm{L}$, así como estudios en Micropterus, donde se demuestra como la adición de eugenol con 5-8 mg/L, es efectivo para mitigar los efectos del transporte en peces, induciendo estado de sedación leve, reduciendo la tasa cardiaca, el volumen sistólico y el gasto cardiaco (Cooke et al. 2004). Al respecto Iversen et al (2009), especula que dentro de los mecanismos de acción del eugenol, esta bloquear la transmisión de la información al hipotálamo y por tanto prevenir la activación del eje hipotálamo.pituitario-interrenal, sin embargo en su estudio en Salmo salar, se observó que concentraciones de eugenol sobre $20 \mathrm{mg} / \mathrm{L}$, pueden bloquear la información y evitar la elevación de corti- sol en plasma durante procedimientos de manejo. Con respecto al balance electrolítico, en el presente trabajo se observó para el tratamiento de $12 \mathrm{~h}$, el sodio y el potasio tuvieron tendencia a disminuir con ambas concentraciones, a pesar que solo fue significativa con el potasio.

En el presente estudio solo se puedo determinar una menor pérdida de potasio con 0.25 y $0.5 \mathrm{mg} / \mathrm{L}$ de eugenol en el agua de transporte del otocinclo, en los dos periodos de transporte, Becker et al (2012) reportó con concentraciones de $1.5 \mu \mathrm{L} / \mathrm{L}$ de eugenol en Rhamdia quelen el menor eflujo neto de sodio, cloro y potasio en transportes de $4 \mathrm{~h}$; esto pudiera explicarse a que el isoeugenol, una molécula estructuralmente similar al eugenol, obstruye los canales de calcio, sodio y potasio e inhibe los receptores D-aspartato (NMDA), cuya función es estimular la apertura de canales iónicos no selectivos y potencializa los receptores GABA A (receptores inhibidores transmembranales del ácido gamma-aminobutírico), modulando la transmisión neural en el cerebro (Aoshima y Hamamoto, 2009) por lo que este anestésico en alguna medida pudiera evitar la pérdida de iones hacia el agua (a favor del gradiente osmótico), tanto por el cierre de canales iónicos como por una posible disminución en permeabilidad branquial ante eventos de estrés.

Es importante tener en cuenta las variabilidad en resultado del anestésico utilizado depende de la especie, procedimientos, factores biológicos, el estadio del ciclo del vida, la edad, peso y tamaño, contenido de lípido, condición física, y condiciones fisicoquímicas del agua, todos estos los factores afectan la tasa metabólica y por tanto la fármacocinética del anestésico (Burka et al.,1997), se espera que no todas las especies respondan de la misma manera.

$\boldsymbol{P}$. axelrodi. presenta una posible reducción en la tasa metabólica en los ensayos de transporte con la adición de eugenol, ya que se disminuyeron significativamente $(\mathrm{P}<0.05)$ los niveles de amonio durante el transporte de $24 \mathrm{~h}$ que no se relaciona con la mortalidad postransporte, similar a estos resultados, Ramirez-Duarte et al, (2010a) en donde reportó en Ancistrus triradiatus, que concentraciones de $0.5 \mathrm{mg} / \mathrm{L}$ disminuyeron significativamente los niveles de amoniaco total y amonio en transportes de $48 \mathrm{~h}$. No se observaron diferencias significativas para la resistencia de estrés con ningún tratamientos, sin embargo, al respecto Barton y Peter et al (1982), mencionan que los anestésicos que previenen la activación de eje HPI a altas concentraciones, no son efectivos a bajas concentraciones, incluso pueden actuar como estresores exacerbando la respuesta del cortisol, de manera similar Zahl et al., 2012, mostró 
que concentraciones de $5.4 \mathrm{mg} / \mathrm{L}$ de isoeugenol (molécula similar al eugenol) en Salmo salar indujeron estrés evidenciado mediante la liberación de cortisol en el agua (Zahl et al. 2010), cabe resaltar que algunos efectos dañinos se han reportado con la exposición a altas concentraciones de eugenol $(30 \mathrm{mg} / \mathrm{L})$ en Oncorhynchus mikyss, por 10 minutos, donde se observó una leve afectación en órganos y tejidos con un incremento en la formación de especies reactivas de oxígeno que conllevo a un daño oxidativo lipídico y a la inhibición de capacidades antioxidantes (Velisek et al. 2011). En los ensayos con cardenal, la pérdida de iones también tuvo tendencia a disminuir con 0.25 y $0.5 \mathrm{mg} / \mathrm{L}$ de eugenol en ambos periodos (con diferencias significativas en E12-0.25; E12-0.5 y E24-0.25 para sodio y E12-0.25 y E24-0.50 para potasio), de manera similar a los ensayos discutidos para Otocinclus $s p$.

De manera general, basándose principalmente en nuestros resultados en el mejoramiento de la mortalidad 7 días post-trasnporte, y la disminución en la pérdida de iones al agua, la adición de $0.5 \mathrm{mg} / \mathrm{L}$ de eugenol en Otocinclus sp., proyecta ser la concentración recomendada para el transporte y para $P$. axelordi por la reducción de amonio en $24 \mathrm{~h}$ de transporte y no tener diferencias con respecto al control en resistencia a soluciones hiperosmóticas postransporte, se recomendaría del mismo modo $0.5 \mathrm{mg} / \mathrm{L}$ de eugenol.

\section{Adición de sal en el transporte}

Debido a que durante los procesos de transporte se activa el sistema del eje neuro-endocrino, lo que resulta en la liberación de cortisol y catecolaminas como respuesta primaria al estrés (Wendelaar-Bonga, 1997), y como respuesta secundaria, se puede detectar alteraciones de glucosa en sangre y electrolitos, principalmente sodio y cloro (Carmichael et al., 1983), con la adición de sal es esperado la reducción del gradiente osmótico entre el pez y los fluidos internos y el agua, estimulando la producción de moco, reduciendo la agitación de los peces en las unidades de trasnporte y reduciendo el estrés en transporte (Tsuzuki et al, 2001).

Otocinclus sp. Con respecto a las variables de calidad de agua, en los ensayos de sal, solo se observó una disminución de nitritos con el tratamiento de sal al 1.5 $\mathrm{g} / \mathrm{L}$, con respecto a $0.5 \mathrm{~g} / \mathrm{L}$ en transportes de $12 \mathrm{~h}$, ninguna otra variable de calidad de agua mostró diferencias con la adición de esta sustancia en ninguno de los dos periodos de transporte. Con respecto a la mortalidad, se observó que hubo una mejoría en la sobrevivencia de los peces postransporte con concentraciones de sal 1.5 y $2.5 \mathrm{~g} / \mathrm{L}$ en comparación con los otros grupos en transportes de $12 \mathrm{~h}$ y un mejoramiento de la mortalidad acumulada 7 días postransporte de $24 \mathrm{~h}$ con $1.5 \mathrm{~g} / \mathrm{L}$ de sal. Ninguno de los grupos presentó diferencias significativas en relación con la resistencia a soluciones hiperosmóticas. Estos resultados son concordantes con los reportados por Ramírez-Duarte et al. (2011), donde la adición de sal con concentraciones de 0.5 y $1 \mathrm{~g} / \mathrm{L}$ al agua de transporte de $48 \mathrm{~h}$ en Ancistrus triradiatus disminuyó significativamente la mortalidad postransporte y la mortalidad acumulada después de 7 días. Sin embargo menciona que exposiciones mayores a $2 \mathrm{~g} / \mathrm{L}$ en este silurido, induce a una mayor mortalidad acumulada 7 días en periodos de 48 h; de igual manera no encontró diferencias significativas en la resistencia a soluciones hiperosmóticas. Con respecto a la pérdida de iones en el agua, con la exposición a sal, el delta de sodio presenta una disminución en todos los tratamientos siendo significativa con 1.5 y $2.5 \mathrm{~g} / \mathrm{L}$ en $12 \mathrm{~h}$, y $0.5 ; 1.5$ y $2.5 \mathrm{~g} / \mathrm{L}$ en 24 $h$, sin embargo es importante tener en cuenta que las mortalidades también disminuyen en tratamientos de 1.5 y $2.5 \mathrm{~g} / \mathrm{L}$, los delta de potasio y cloruro disminuyen con las más bajas concentraciones de sal, pero fluctúan aumentando con tratamientos con 1.5 y $2.5 \mathrm{~g} / \mathrm{L}$ y disminuyendo en el delta de potasio con 1.5 y $2.5 \mathrm{~g} / \mathrm{L}$ en $24 \mathrm{~h}$; al respecto se han reportado niveles estables en el suero de sodio y cloro en matrinxã con $6 \mathrm{~g} / \mathrm{L}$ por transporte de $4 \mathrm{~h}$ (Carneiro y Urbinati, 2001), mientras que Ramírez-Duarte et al (2011), observó una disminución en la conductividad en el agua con 0.5 y 1 g/L de sal, en Ancistrus triradiatus. Bajas concentraciones han evidenciado la contribución al balance electrolítico, para el presente caso, se observa una disminución del sodio que no se puede asociar solo a la menor pérdida por un equilibrio electrolítico de los peces por inferencia de la mortalidad (que repercutiría en una mayor pérdida de iones en el agua), se desconoce la razón de las fluctuaciones de la concentración de cloruros y potasio con respecto a las concentraciones 1.5 y $2.5 \mathrm{~g} / \mathrm{L}$, ya que se esperaría que un ambiente aparentemente dentro o incluso sobre el rango de salinidad que maneja la especie, los iones tenderían al balance (si estuviese sobre el rango de salinidad de la especie) o incluso una hipo-osmoregulación donde vendría a cabo invasión de iones (para el caso una pérdida de iones en el agua) y deshidratación (Baldiseroto el at; 2007).

La concentración de $1.5 \mathrm{~g} / \mathrm{L}$ de sal para esta especie ejerció un mejor efecto que el resto de las concentraciones utilizadas, debido al mejoramiento de la mortalidad postransporte en $12 \mathrm{~h}$ y mortalidad acumulada 7 días en $24 \mathrm{~h}$.

P. axelrodi. esta sustancia, mostró a nivel de calidad de agua, disminuciones significativas en los valores de $\mathrm{pH}$ 
y las concentraciones de nitrito, con la adición de 0.25 y $0.5 \mathrm{~g} / \mathrm{L}$ en transporte de $12 \mathrm{~h}$. Durante el transporte de $24 \mathrm{~h}$, concentraciones los niveles de amonio fueron menores en el agua de transporte, aunque puede estar relacionada con una menor mortalidad postransporte en este tratamiento. La incorporación de 1.5 y $2.5 \mathrm{~g} / \mathrm{L}$, presenta mejor tasa de disminución del número de peces que pierden el eje de nado transportados por 12 h, sin embargo con $2.5 \mathrm{~g} / \mathrm{L}$, el $50 \%$ de la población de estos peces perdieron el eje de nado con más velocidad que los demás tratamientos y control, por lo que no se puede afirmar que tuvieron la mejor respuesta a la condiciones de estrés osmótico en este caso; al respecto, Oyoo-okoth et al. (2011), reportó que concentraciones desde 0.5 a 8 UPS (unidades prácticas de salinidad), no presenta correlaciones negativas con los niveles de cortisol en plasma, glucosa y amonio en Labeo victorians en transporte de $6 \mathrm{~h}$, por lo que no ejercen una menor resistencia al estrés durante el transporte. Las tres concentraciones utilizadas en los ensayos, otorgaron una mejor sobrevivencia posterior al desempaque, mientras que las concentraciones de 1.5 y $2.5 \mathrm{~g} / \mathrm{L}$, fueron mejores para mejorar sobrevivencia luego de 7 días postransporte para el periodo de $12 \mathrm{~h}$; el incremento en los niveles de sobrevivencia de esta especie se presenta de manera similar al mejoramientos en sobrevivencia en Otocinclus sp (presente estudio), A. triradiatus (Ramirez-Duarte el al, 2011) y Carassius auratus (Luz et al., 2008) con la adición de bajas concentraciones de sal. Para el transporte de $12 \mathrm{~h}$, los iones cloro sodio y potasio para esta especie si tienden a disminuir con la adición de sal en todos los casos, siendo significativo en cloruro en todos con todos los tratamientos, para potasio con concentraciones de $2.5 \mathrm{~g} / \mathrm{L}$ en 12 h. En 24 h la concentración de iones es más fluctuante y heterogénea, por lo que se considera que las variaciones en mortalidad pudieron influir en este comportamiento.

En esta especie, la concentración de $1.5 \mathrm{~g} / \mathrm{L}$ de sal otorgó además de la mejor resistencia a la solución hiperosmótica, mejor sobrevivencia posterior al transporte y mortalidad acumulada después de 7 días, la pérdida de iones al agua fue fluctuante en los diferentes tratamientos.

\section{Adición de carbonato de calcio en el transporte}

Otocinclus sp. Para esta especie esta sustancia no representó en general efectos benéficos sobre la calidad del agua durante el transporte, por el contrario la adición de $0.5 \mathrm{~g} / \mathrm{L}$ fue deletéreo, ya que provocó mayor excreción de amonio en transportes de $12 \mathrm{~h}$, (no relacionado con la mortalidad ya que disminuyó en este caso), la misma tenencia ocurrió con los niveles de nitrito en 24 h, con la concentración de 1 $\mathrm{g} / \mathrm{L}$, este si relacionándose con el incremento en mortalidad. Con respecto a la pérdida de iones al agua, el cloruro y sodio tienden a la disminución con concentraciones de 0.5 y $1 \mathrm{~g} / \mathrm{L}$ (no relacionados con la mortalidad postransporte), sin diferencias para el potasio, lo que es consistente con la acción del calcio, a través de reacciones aniónicas puede alterar la hidratación de estructuras orgánicas sobre las branquias y reducir su permeabilidad al sodio y el cloro (Potts, 1984). No se observaron efectos significativos de la adición de esta sustancia sobre la resistencia al estrés, contrario a lo reportado por Bendhack and Urbinati (2009), que demostraron que concentraciones bajas de 150 y $300 \mathrm{mg} / \mathrm{L}$ disminuyen la concentración de cortisol en sangre. Aunque no se observó diferencias en la sobrevivencia postransporte, si fue mejor la mortalidad postransporte acumulada luego de 7 días con la adición de $0.1 \mathrm{~g} / \mathrm{L}$ durante las $12 \mathrm{~h}$ de transporte. Similar a lo reportado para Morone saxatilis y Morone chrysops con la adición de calcio en el agua (Grizzle et al., 1985).

P. axelrodi. En los experimentos con carbonato de calcio, las variables de calidad de agua no presentan claras evidencias de mejoramiento, la única variable en el agua que se vio afectada, fue potasio con la menor nivel, en el tratamiento de $0.1 \mathrm{~g} / \mathrm{L}$, para el transporte de $12 \mathrm{~h}$, mientras que para el transporte de $24 \mathrm{~h}$ con esta misma concentración, el delta del ion aumenta de manera significativa.

Para la prueba de estrés en transporte de $12 \mathrm{~h}$, la adición de $0.1 \mathrm{~g} / \mathrm{L}$ fue la que mejor resultados obtuvo, en contraste con la adición $1 \mathrm{~g} / \mathrm{L}$, en donde aumentó el número de peces que perdió el eje de nado. En $24 \mathrm{~h}$ el resultados no mostraron una mejoría en la resistencia al estrés, incluso el tratamiento con $1 \mathrm{~g} / \mathrm{L}$, la afecta de manera negativa en comparación con las otras concentraciones, con una tasa de disminución significativamente mayor. La mortalidad para este caso solo fue mejorada con la concentracion de $0.1 \mathrm{~g} / \mathrm{L}$ en el ensayo de $24 \mathrm{~h}$ únicamente.

Un aspecto para resaltar es la precipitación de las grandes partículas del carbonato industrial, incluso a la más baja concentración no se solubiliza completamente, dejando en duda la real concentración en el agua, a pesar de esto para ambas especies aparentes concentraciones de $0.1 \mathrm{mg} / \mathrm{L}$ ejercieron un efecto benéfico en incremento a soluciones hiperosmóticas y aumento de la sobrevivencia en trasnportes de $P$. axelrodi y solo para el mejoramiento de la sobrevivencia en Otocinclus $s p$. De manera general esta sustancia fue la que 
resultó en un menor mejoramiento de las variables evaluadas, por lo que se recomendaría el uso de sal y eugenol antes que de carbonato de calcio industrial para estas especies.

\section{Agradecimientos}

Al Instituto de Acuicultura de los Llanos IALL, al Grupo de Investigación en Sanidad de Organismos Acuáticos, a Fredy Armando Aguilar por sus valiosas contribuciones y asesoría estadística y finalmente al Instituto de Investigaciones de la Orinoquia IIOC, por el apoyo financiero para la realización del presente trabajo.

\section{Referencias}

Aoshima H, Hamamoto K. Potentiation of GABAA receptors expressed in Xenopus oocytes by perfume and phytoncid. Biosciense Biotechnology and Biochemistry, 1999; 63 (4): 743-748

Baldiceroto B, Mancera J, Kapoor BG. 2007. Fish osmoregulation. Osmoregulation and fish transportation. Science Publishers, Enfield NH USA: p 235.

Barton BA, Peter RE. Plasma cortisol stress response in fingerling rainbow trout (Salmo gairdneri Richardson), to various transport conditions, anaesthesia, and cold shock. Journal Fish Biology, 1982; 20: 39- 51

Becker A, Parodi TV, Heinzmann BM, Baldisserotto B. Transportation of silver catfish, Rhamdia quelen, in water with eugenol and the essential oil of Lippia alba. Fish Physiology and Biochemistry, 2012;38: 789-796

Bendhack F, Urbinati EC. Mitigating stress effects during transportation of matrinxã (Brycon amazonicus Günther, 1869; Characidae) through the application of calcium sulfate. Journal of Applied Ichthyology, 2009; 25(2): 201-205

Blanco MC. 1992. Ordenamiento de las pesquerías de los peces ornamentales en los Llanos Orientales. Informe técnico. INDERENA - Instituto Nacional de Recursos Naturales Renovables. Bogotá, Colombia.

Bower C, BidwelL J. Ionization of ammonia in sea water effects of temperature, $\mathrm{pH}$, and salinity on Salmo salar L. Journal of Fisheries Research Board of Canada, 1978; 35: 1012-1016

Burka JF, Hammel KL, Horsberg TE, Johnson GR, Rainnie DJ, Spears DJ. Drugs in salmonid aquaculture-a review. Journal of Veterinary Pharmacology and Therapeutics, 1997; 20: 333-349

Carmichael GJ, Tomasso JR, Simco BA, Davis KB. Characterization and alleviation of stress associated with hauling largemouth bass. Transaction of the American Fisheries Society, 1983; 113: 778-785

Carneiro PCF, Urbinatie C. Salt as a stress response mitigator of matrinxã, Brycon cephalus, during transport. Aquaculture Research, 2001; 32: 297-304

Cooke SJ, Suski CD, Ostrand KG, Trufts BL, Wahl DH. Behavioral and physiological assessment of low concentrations of clove oil anaesthetic for handling and transporting largemouth bass (Micropterus salmoides). Aquaculture, 2004; 239(1-4): 509-529

Gomes L, Araujo-Lima C, Roubach R, Urbinati E. Avaliação dos efeitos da adição de sal e da densidade no transporte de tambaqui. Pesquisa agropecuaria brasileira, 2003; 38: 283-290

Grizzle JM, Mauldin AC, Young D, Henderson E. Survival of juvenile striped bass (Morone saxatilis) and Morone hybrid bass (Morone chrysops $x$ Morone saxatilis) increased by addition of calcium to soft water. Aquaculture, 1985; 46:167-171

Kioshi Aoki Inoue LA, Afonso LOB, Iwama GK, Moraes G. Effects of clove oil on the stress response of matrinxã (Brycon cephalus) subjected to transport. Acta Amazonica, 2005; 35(2): 289-295

Iversen M, Eliassen RA, Finstad B. Potential benefit of clove oil sedation on animal welfare during salmon smolt, Salmo salar L. transport and transfer to sea. Aquaculture Research, 2009; 40: 233-241

Johnson S. K. Transport of live fish. Aquaculture Management 1979; 5: $20-24$

Johanson $\mathrm{O}$, Wedborg $\mathrm{M}$. The ammonia-ammonium equilibrium in the seawater at temperature between 5 and $25^{\circ} \mathrm{C}$. Journal of Solution Chemistry 1980; 9: 37- 44

Kildea MA, Allan GL, Kearney RE. Accumulation and clearance of the anaesthetics clove oil and AQUI-S(TM) from the edible tissue of silver perch (Bidyanus bidyanus). Aquaculture, 2004; 232: 265-277

Lim LC, Dhert P, Sorgeloos P. Recent developments and improvements in ornamental fish packaging systems for air transport. Aquaculture Research 2003; 34: 923-935

Luz RK, Martínez-Álvarez RM, De Pedro N, Delgado MJ. Growth food intake regulation and metabolic adaptations in goldfish (Carassius auratus) exposed to different salinities. Aquaculture, 2008; 276: 171-178

Mazik PM, Simco BA, Parker NC. Influence of Water Hardness and Salts on Survival and Physiological Characteristics of Striped Bass during and after Transport. Transactions of the American Fisheries Society, 1991; 120: 121-126

MADR -Ministerio de Agricultura y CCl -Desarrollo Rural y Corporación Colombiana Internacional. 2010. Pesca y Acuicultura Colombia 2009 "Informe técnico regional de cuencas del Orinoco y Amazonas". Corporación Colombiana Internacional Bogotá Colombia

Elijah O, Cherop L, Ngugi CC, Chepkirui-Boit V, Manguya-Lusega V, Sabwa JA, Charo-Karisa J. Survival and physiological response of Labeo victorianus (Pisces: Cyprinidae, Boulenger 1901) juveniles to transport stress under a salinity gradient. Aquaculture, $2011 ; 319(1-2): 226-231$

Paternoster R, Brame R, Mazerolle P, Piquero A. Using the correct statistical test for the equality of regression coefficients. Criminology, 1998; 36 (4): 859-866

Pineda AIZ. 1996. Investigación de pesquerías en la Orinoquía Colombiana: Evaluación biológico-pesquera de las principales especies de consumo en Arauca y manejo y evaluación de peces 
ornamentales. Instituto Nacional de Pesca y Acuicultura INPA, Regional Oriental. Arauca, Colombia

Potts WTW. 1984. Transepithelial potentials in fish gills. In: Ion and Water Transfer, Hoar WS, Randall DJ, Brett JR. (eds.). Academic Press, Orlando, pp. 105-128.

Ramírez GH, Ajiaco MRE. 2001. La pesca en la baja Orinoquía colombiana: una visión integral. MADR, PRONATA, Colciencias, INPA. Colombia. www.Proexport.com.co.2004(http://www. oie.int/esp/normes/fcode/es_sommaire.htm).

Ramírez-Duarte WF, Pineda-Quiroga C, Martínez N, Eslava-Mocha P. 2010a. Comparación del efecto del cloruro de sodio, zeolita y eugenol en el transporte de Ancistrus triradiatus. In: Instituto de Acuicultura, U.d.I.L. (Ed.), XVI Jornada de Acuicultura, Villavicencio, Meta (Colombia), pp. 107-119

Ramírez-Duarte WF, Pineda-Quiroga C, Martínez N, Eslava-Mocha P. R. 2010b. Evaluación de diferentes concentraciones de sal en el transporte de loricaridos. In: Instituto de Acuicultura, U.d.I.L. (Ed.), XVI Jornada de Acuicultura, Villavicencio, Meta (Colombia), pp. 98-106

Ramírez-Duarte WF, Parada S, Martínez N, Eslava-Mocha PR, Figueroa J, Iregui C. 2009. Manejo de peces de la familia Loridariidae en el municipio de Acacías, Meta: monitoreo desde la captura hasta la comercialización (Reporte preliminar). Instituto de Acuicultura, U.d.I.L. (Ed.), XV Jornada de Acuicultura, Villavicencio, Meta (Colombia), pp. 58-65.

Ramírez-Duarte WF, Pineda-Quiroga C, Martínez N, Eslava-Mocha PR. Use of sodium chloride and zeolite during shipment of An- cistrus triradiatus under high temperature. Neotropical Ichthyology, 2011; 9(4): 909-914

Tsuzuki MY, Ogawa K, Strussmann CA, Maita M, Takashima F. Physiological response during stress and subsequent recovery in adult pejerrey Odontesthes bonariensis. Aquaculture, 2001; 200: $349-362$

Velisek J, Azbeta S, Zhi-Hua L, Sarka S, Jan T. Comparison of the effects of four anaesthetics on blood biochemical profiles and oxidative stress biomarkers in rainbow trout. Aquaculture, 2011; 310(3-4): 369-375

Weirich CR, Tomasso JR. Confinement- and transport-induced stress on red drum juvenile: effect of salinity. The Progressive Fish-Culturist , 1991; 53: 146-149

Weirich CR, Tomasso JR. Confinement- and transport-induced stress in White Bass Morone chrysops x Striped Bass M. saxatilis Hybrids: Effects of Calcium and Salinity. Journal of the World Aquaculture Society, 1992; 23(1): 49-57

Wendelaar Bonga SE. The stress response in fish. Physiologycal Reviews 1997; 77: 591-625

Zahl IH, Kiessling A, Samuelsen OB, Olsen RE. Anesthesia induces stress in Atlantic salmon (Salmo salar), Atlantic cod (Gadus morhua) and Atlantic halibut (Hippog/ossus hippoglossus). Fish physiology and Biochemistry, 2010; 36(3): 719-30 